\title{
1-Nitropyrene Exposure as Genotoxicity and Oxidative Stress Biomarker
}

\author{
Jayfe Anthony Abrea ${ }^{1}$ \\ ${ }^{1}$ University of San Carlos
}

August 6, 2020

\section{Introduction}

Belonging to the group of nitrated polycyclic aromatic hydrocarbons (NPAH), this 1-nitropyrene (1-NP) molecule, shown in Figure 1, often comes out as a dominant by-product of incomplete combustions from coal, diesel engines from mobile and stationary sources, cigarette smoke, cooked meat products and biomass burning. Aside from its dispersion in the atmosphere with concentrations arriving up to $57 \mathrm{pg} / \mathrm{m}^{3}$ in the air over urban and suburban areas, these hydrocarbons can deposit to ambient fine particulate matter (PM 2.5) which can spread towards inland bodies of water to be accumulated by aquatic species and then also reach towards human health mainly by inhalation, thereby posing respiratory risks. In fact, it has been listed as an International Agency for Research on Cancer (IARC) Group 2A carcinogen which indicates probable carcinogenicity and high respiratory health risk to humans [1].

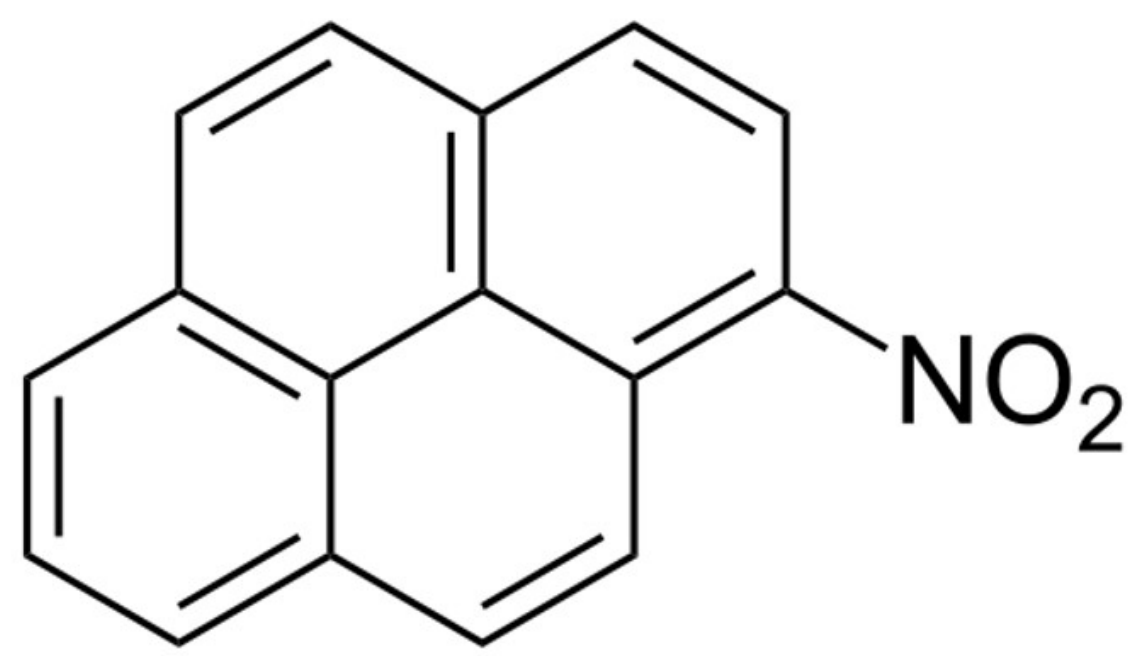

Figure 1: Structure of 1-nitropyrene.

This predominant NPAH molecule can also pollute the aquatic ecosystems which poses genotoxic effects, specifically mutagenic, and oxidative stress to sub-organism levels of biological organization like fishes. Several researches have pointed out that the accumulation of 1-nitropyrene is a possible bioindicator of oxidative stress and genotoxicity among several fish species (e.g. red sea bream, marbled flounder, and tilapia) and 
as well as of aquatic pollution evaluation. This paper describes the related researches of the environmental 1-nitropyrene exposure as bioindicator oxidative stress and genotoxicity.

\section{Detections and Their Principles}

Evaluation of bioindicator to oxidative stress and genotoxicity can be conducted with an experiment on a representative fish organism in the locality, where different concentrations and different exposure time of 1-nitropyrene are set. Blood samples are analyzed for its genotoxic effects via micronuclei (MN) analysis, nuclear abnormality (NA) analysis, and DNA oxidation analysis. Liver samples are analyzed for its activities on glutathione peroxidase and assays on lipid peroxidation and carbonyl protein. Concentrations of 1-NP in the aqueous environment are analyzed using high performance liquid chromatography (HPLC) with massspectrometry detection.

\section{Micronuclei (MN) and Nuclear Abnormality (NA) Analysis}

These tests are used for detecting chromosomal damage. Micronuclei are ovoid or circular bodies containing fragmented or whole chromosomal material that separate from the nuclei. Their presence reflects the structural and/or numerical chromosomal aberrations arising during mitosis [2]. The nuclear abnormality test serves as a compliment to MN test which has been used as an evaluation to cytogenetic damage in fish species. The abnormalities present in erythrocytes can be classified as notched, lobed, blebbed, and vacuolated, in addition to the typical micronuclei [3].

\section{Assays of Oxidative-Damaged Target}

The continuous efflux of reactive oxygen species (ROS), which may be in radical and radical form with high reactivity, from endogenous, where 1-NP belongs, and exogenous sources results in continuous and accumulative oxidative damage to cellular components and alters many cellular functions. Most of the targets that are vulnerable to these attacks are proteinaceous enzymes, lipidic membranes, and DNA as displayed in a series of chain reactions and target attacks shown in Figure 2 [4]. The degree of damage on each target by 1-NP exposure can be assessed with proper instrumentation and biochemical analyses. 


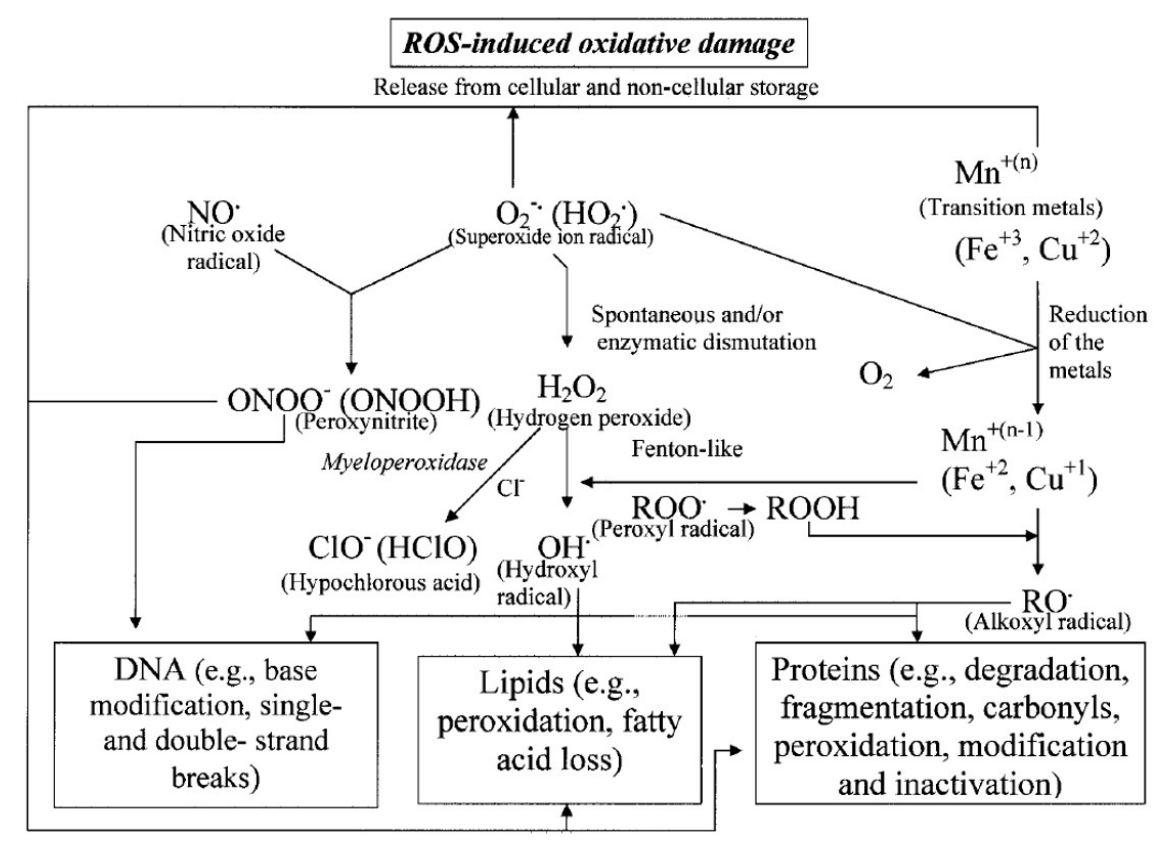

Figure 2: Reactive oxygen species (ROS)-induced oxidative damage pathway taken from [5].

\section{a. DNA Oxidation Analysis}

Most of the attacks on the stable DNA come from the hydroxyl radical, $\mathrm{OH}$. The $\mathrm{OH}$ can attack guanine at its C-8 position to yield an oxidation product, 8-hydroxydeoxyguanosine (8-OHdG), which can be quantified using a competitive enzyme-linked immunosorbent assays (ELISA) kit (New 8-OHdOG Check, Japan Institute for the Control of Aging, Japan). The underlying principle for these immunoassays is based on highly specific interaction of the target PAH analyte with a corresponding antibody, followed by the production of a colorimetric/fluorometric response to allow quantitative determination of 1-NP. Figure 3 summarizes the mechanism of ELISA.

\section{b. Lipid Peroxidation Analysis}

Lipid peroxidation reflects a chain reaction between polyunsaturated fatty acids and ROS producing lipid peroxides and hydrocarbon polymers that are both highly toxic to the cell. Malonyldialdehyde (MDA) is an end product of peroxidation of polyunsaturated fatty acids and related esters. MDA is spectrophotometrically quantified by reacting with thiobarbituric acid (TBA), forming a colored complex with a maximum absorbance at $532 \mathrm{~nm}[7]$. 


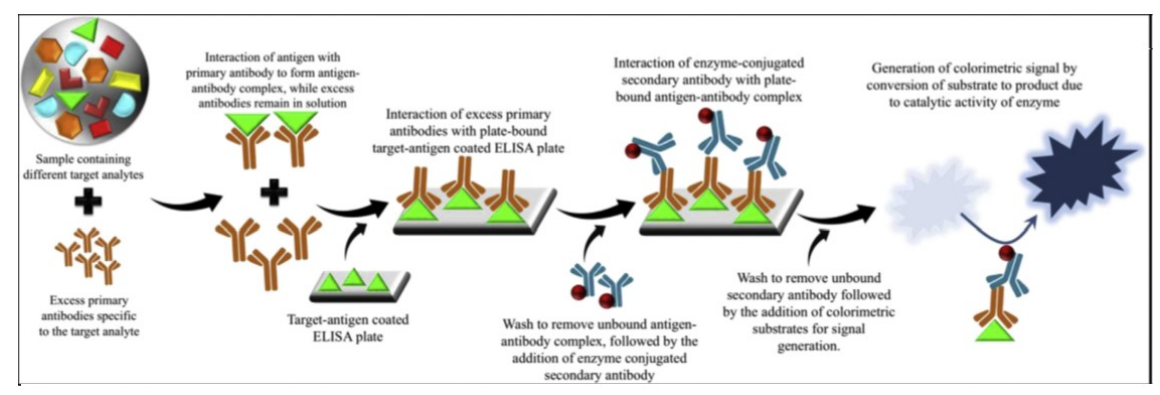

Figure 3: Schematic representation of a competitive enzyme-linked immunosorbent assay. Taken from [6].

\section{c. Carbonyl Protein Assay}

Carbonyl protein content is important in determining the extent of oxidation towards the protein backbones and amino acid residues such as proline, arginine, lysine, and threonine by ROS molecules. Protein oxidation leads to loss of critical sulfhydryl groups in addition to modification of amino acids, leading to the formation of carbonyl and other oxidized moieties. The oxidized proteins can be quantified using the 2,4-dinitrophenylhydrazine (DNPH). DNPH reacts with carbonyl proteins, forming a Schiff base to produce dinitrophenylhydrazone products, the levels of which can be analyzed spectrophotometrically at $375 \mathrm{~nm}$ and correlated to the levels of oxidized proteins [8].

\section{Glutathione Peroxidase Activity}

The presence of ROS can be countered by the production of antioxidants in our body through a series of redox reactions, forming what we call as redox homeostasis. This balance is disturbed by the excessive production of free radicals and ROS that have deleterious effects on our body functions. Thus, quantifying a specified antioxidant's amount in an organism is a measure of the oxidative stress extent. One of these is the glutathione peroxidase (GPx), an enzyme that catalyzes the reduction of hydrogen peroxide and lipid peroxides to water and their corresponding lipid alcohols via the oxidation of reduced glutathione (GSH) into glutathione disulfide (GSSG). Activity was determined spectrophotometrically by coupling the oxidation of glutathione and NADPH using glutathione reductase (GR). GPx catalyzes the oxidation of glutathione by cumene hydroperoxide (for selenium-independent GPx) or hydrogen peroxide (for selenium-dependent GPx). The oxidized glutathione is later reduced by exogenous glutathione reductase causing the coenzyme of the reaction, NADPH, to become oxidized into NADP + . The change in the absorbance can then be read spectrophotometrically at $\lambda=340 \mathrm{~nm}$ [9].

\section{Analysis of 1-NP}

There's a need for 1-NP to be separated out from the bound body tissues and due derivatization before it is injected to high performance liquid chromatography (HPLC). HPLC is an important qualitative and quantitative technique, generally used for the estimation of pharmaceutical and biological samples thru proper separation. Depending on the molecular size and polarity effect, 1-NP moves differently from the rest of the molecules in the stationary phase when the mobile phase is being passed through. Thus, optimization should be set first to maximize sensitivity and selectivity of its resulting chromatogram [10]. This is then detected using mass spectrometry wherein the instrument generates multiple ions from the sample under investigation, it then separates them according to their specific mass-to-charge ratio $(\mathrm{m} / \mathrm{z})$, and then records 
the relative abundance of each ion type. In a setup by Bacolod, et. al., detection limit (LOD) of 1-NP was $2 \mathrm{ng} / \mathrm{kg}$ in fish and $0.002 \mathrm{ng} / \mathrm{L}$ in water with recoveries from 95 to $98 \%$ [11].

Separation of 1-NP can be done by thorough extraction of its freeze-dried homogenized soft tissues with $3: 1(\mathrm{v} / \mathrm{v})$ dichloromethane-methanol prior to the addition of hexane-water mixture to the liquid extract. Significant amounts of 1-NP are transferred towards the hexane layer during successive extraction. The dehydrated hexane layer containing 1-NP is loaded into silica gel column, which serves as the stationary phase, where the target chemicals are eluted with the mobile phase in the form of $60 \%$ acetone-hexane (v/v). Eluate was spiked with $100 \mu \mathrm{L}$ of $100 \mathrm{ng} / \mathrm{mL}$ 1-nitropyrene- $\mathrm{d}_{9}$ as an internal standard before changing solvent to $1.5 \mathrm{~mL}$ of ethanol. Derivatization was conducted by reduction with $3 \mathrm{~mL} 20 \% \mathrm{NaHS}$ at $95{ }^{\circ} \mathrm{C}$ for $60 \mathrm{~min}$ and acetylation with acetic acid anhydride at $65{ }^{\circ} \mathrm{C}$ for $45 \mathrm{~min}$. The acetylated 1-NP was extracted with $10 \mathrm{~mL}$ dichloromethane and the extract was evaporated to dryness under a gentle nitrogen stream. The residue was dissolved in $0.1 \mathrm{~mL}$ of $80 \%$ acetonitrile. The resulting 1-NP solution was measured using highperformance liquid chromatography with mass spectrometry detector in positive electron scanning ionization mode.

\section{Biomarker Induction}

In the study by Bacolod, et. al., there's an observed positive correlations ( $\mathrm{r}>0.49$ ) between the 1NP concentration exposure of the Oreochromis niloticus to the levels of thiobarbituric reactive substances (TBARS), GPx, carbonyl protein, 8-OHdG, MN and NA which suggests occurrence of oxidative stress and genotoxicity. Increase of 8-OHdG in the fish serum served to be the primary toxic effect of the 1-NP exposure thus increasing DNA lesions are then expected for this organism. Generally, 1-NP induces the ROS that attacks the target sites especially in DNA. However, intracellular levels of 8-OHdG may be initially hindered due to the presence of 8-OHdG repair enzymes. In the study by Kim, et. al., results suggested that the repair mechanism of human oxoguanine glycosylase (hOGG1) and AP endonuclease (APEX) prevented an 8-OHdG formation increase in cellular DNA of A549 human lung adenocarcinoma cell line below $250 \mu \mathrm{M} 1-\mathrm{NP}$, and that excessive generation of ROS exceeds the capacity for DNA repair, resulting to slight increase in 8-OHdG [12]. Thus, it is imperative that the lack of significant 8-OHdG level increase for a certain organism in an increasing 1-NP exposure would not necessarily conclude the absence of DNA oxidative damage.

When 1-NP is metabolized inside the organism, electrophilic hydroxyl amino metabolites are formed wherein it transforms into DNA adducts, the primary form of which is N-(deoxyguanosin-8-yl)-1-aminopyrene (dG-C8-AP), as shown in Figure 4. The production of dG-C8-AP has been observed to be present in several in vivo and in vitro studies. DNA damage induced by the metabolites of 1-nitropyrene produced mutations, primarily frameshifts in bacterial systems and base substitutions (mostly GC-TA) in mammalian cells. Standard slippage models explained the frameshifts, and polymerase misincorporation models explained the base substitutions. In addition to gene mutation, 1-nitropyrene also caused chromosomal mutation, such as micronucleus formation, and morphological cell transformation. In contrast to the mutation spectrum of 1-nitropyrene in mammalian cells in vitro or in bacterial cells, oncogenes in 1-nitropyrene- induced tumors in rodents have primarily AT-GC mutations, as well as altered patterns of expression [13]. In general, 1NP not only induced oxidative stress and the formation of reactive oxygen species, but also inflammatory proteins and apoptosis in mammalian cell systems and rodents. These mechanisms, together with its direct genotoxicity, could contribute to the carcinogenicity of 1-nitropyrene. 
<smiles>CC(C)Nc1ccc2ccc3cccc4ccc1c2c34</smiles>

N-hydroxy-1-aminopyrene 1-nitropyrene

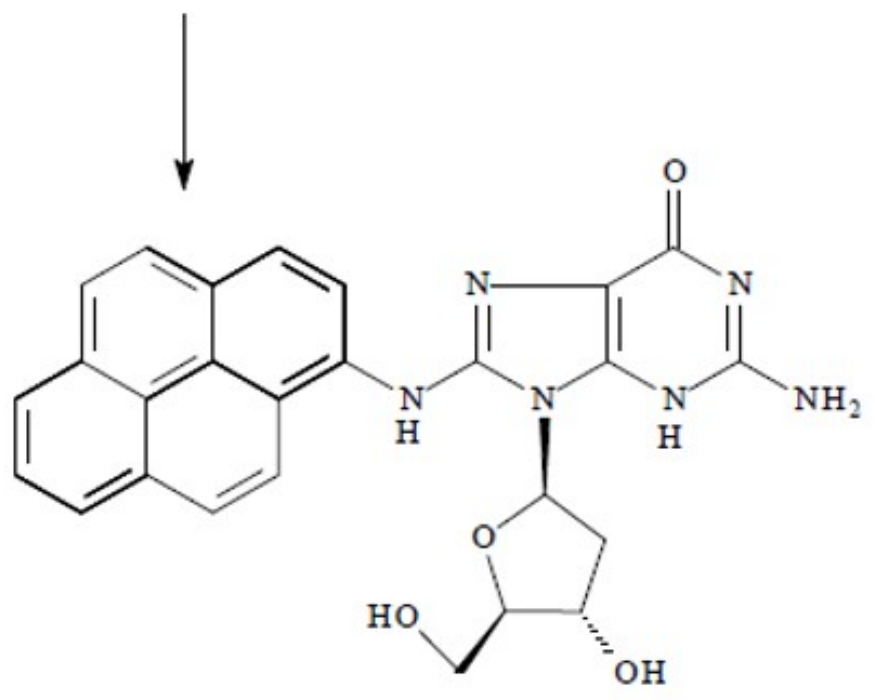

dG-C8-AP

dG-C8-AP, $N$-(deoxyguanosin-8-yl)-1-aminopyrene

Figure 4: Induction of DNA Adduct with 1-NP.

\section{References}

[1] IARC, 2016. Agents Classified by the IARC Monographs 1-115. pp. 10.

[2] Al-Sabti, K., \& Metcalfe, C. D. (1995). Fish micronuclei for assessing genotoxicity in water. Mutation Research/Genetic Toxicology, 343(2-3), 121-135. https://doi.org/10.1016/0165-1218(95)90078-0

[3] Carrasco, [3] Carrasco, K. R., Tilbury, K. L., \& Myers, M. S. (1990). Assessment of the Piscine Micronucleus Test as an in situ Biological indicator of Chemical Contaminant Effects. Canadian Journal of Fisheries and Aquatic Sciences, 47(11), 2123-2136. https://doi.org/10.1139/f90-237

[4] Kohen, R., \& Nyska, A. (2002). Invited review: Oxidation of biological systems: oxidative stress phe- 
nomena, antioxidants, redox reactions, and methods for their quantification. Toxicologic Pathology, 30(6), 620-650. https://doi.org/10.1080/01926230290166724

[5] Kohen, R., \& Nyska, A. (2002). Invited review: Oxidation of biological systems: oxidative stress phenomena, antioxidants, redox reactions, and methods for their quantification. Toxicologic Pathology, 30(6), 620-650. https://doi.org/10.1080/01926230290166724

[6] Behera, B. K., Das, A., Sarkar, D. J., Weerathunge, P., Parida, P. K., Das, B. K., Thavamani, P., Ramanathan, R., \& Bansal, V. (2018). Polycyclic Aromatic Hydrocarbons (PAHs) in inland aquatic ecosystems: Perils and remedies through biosensors and bioremediation. Environmental Pollution, 241, 212233. https://doi.org/10.1016/j.envpol.2018.05.016

[7] Chauhan, A., Chauhan, V., Brown, W. T., \& Cohen, I. (2004). Oxidative stress in autism: Increased lipid peroxidation and reduced serum levels of ceruloplasmin and transferrin - the antioxidant proteins. Life Sciences, 75(21), 2539-2549. https://doi.org/10.1016/j.lfs.2004.04.038

[8] Levine, R. L., Garland, D., Oliver, C. N., Amici, A., Climent, I., Lenz, A.-G., Ahn, B.-W., Shaltiel, S., \& Stadtman, E. R. (1990). [49] Determination of carbonyl content in oxidatively modified proteins. In Methods in Enzymology (Vol. 186, pp. 464-478). Elsevier. https://doi.org/10.1016/0076-6879(90)86141-H

[9] Gallo, G., \& Martino, G. (2009). Red blood cell glutathione peroxidase activity in female nulligravid and pregnant rats. Reproductive Biology and Endocrinology, 7(1), 7. https://doi .org/10.1186/1477-7827-77

[10] Malviya, R., Bansal, V., Pal, O. P., \& Sharma, P. K. (2010). High performance liquid chromatography: a short review. Journal of global pharma technology, 2(5), 22-26.

[11] Bacolod, E. T., Uno, S., Villamor, S. S., \& Koyama, J. (2017). Oxidative stress and genotoxicity biomarker responses in tilapia (Oreochromis niloticus) exposed to environmental concentration of 1nitropyrene. Marine Pollution Bulletin, 124(2), 786-791. https://doi.org/10.1016/j.marpolbul.2017. 01.077

[12] Kim, Y., Ko, Y., Kawamoto, T., \& Kim, H. (2005). The effects of 1-nitropyrene on oxidative DNA damage and expression of DNA repair enzymes. Journal of Occupational Health, 47(3), 261-266. https: //doi.org/10.1539/joh.47.261

[13] IARC, 2012. Diesel and gasoline engine exhausts and some nitroarenes. IARC Monogr. Eval. Carcinog. Risks Hum. 105, 666-676 